IMAGING

\section{High-resolution imaging of the murine placenta}

De Clerca, K. et al. Proc. Natl Acad. Sci. USA 116, 13927-13936 (2019)

Gene knockout $(\mathrm{KO})$ is a common strategy to assess gene function. The ongoing effort of the International Mouse Phenotyping Consortium to generate and phenotypically characterize $5000 \mathrm{KO}$ mouse lines has shown that one third of gene $\mathrm{KO}$ results in embryonic lethality, indicating that these genes are essential for embryogenesis. However, the possible contribution of placental defects to embryonic lethality should also be investigated to avoid misinterpretation of gene function in the developing embryo. Conventionally, defects in placenta formation are assessed by histology. A new study describes the use of a contrast-enhanced microfocus computed tomography (CE-CT)-approach as a new tool with complementary value to histology to quantify placental parameters and to study placental development and pathologies.

\section{AGING}

\section{Telomeres and lifespan}

Whittemore, K. et al. Proc. Natl Acad. Sci. USA 116

15122-15127 (2019)

Although previous studies suggest that telomeres are involved in aging, no correlation has been found between the lifespan of a species and initial telomere length. Humans for example have shorter telomeres than mice $(5-15 \mathrm{~kb}$ versus $50 \mathrm{~kb}$ ) but have much longer lifespans. A new study used highthroughput quantitative fluorescence in situ hybridization (HT Q-FISH) to measure at different ages telomere length in peripheral blood mononuclear cells from individuals of different species of birds and mammals (including mouse, goat, American flamingo, and Sumatran elephant), and calculate telomere shortening rate for each species. The results show that telomere shortening rate, but not initial telomere length, is a powerful predictor of species lifespan.

$A L B$

https://doi.org/10.1038/s41684-019-0388-5

\section{IMAGING}

\section{ANIMAL BEHAVIOR}

\section{TDAG51 linked to postpartum depression}

Yun, H. et al. PLoS Genet. 15, e1008214 (2019)

Postpartum depression is a severe mental disorder that affects $10-15 \%$ of women during pregnancy or after giving birth. The biological risk factors of postpartum depression - notably the influence of genetic risk factors - are not well identified, whereas many psychosocial factors, such as a history of psychiatric illness, have been studied in detail. By showing that the survival of pups born to TDAG51 $1^{-/-}$dams is reduced after parturition compared with wild-type mice, and that TDAG51 $1^{-/-}$dams have enhanced susceptibility to depressivelike behavior, a new study supports the protective role of $\mathrm{T}$ cell death-associated gene 51 protein (TDAG51) - a transcription factor highly expressed in the brain and regulated by stimuli, such as injury, infection and stress - against maternal care defects and depressive-like behavior after parturition.

\section{A sensor for $\mathrm{GABA}$}

Marvin, J.S. et al. Nat. Methods 16, 763-770(2019)

Although GABA ( $\gamma$-aminobutyric acid) is the primary inhibitory neurotransmitter in the mammalian central nervous system, methods for directly imaging GABAergic signaling are lacking. Now, a study reports the development of an intensity-based GABA sensing fluorescence reporter (iGABASnFR) to track GABA transient in cultured neurons, mouse brain slices, zebrafish, and in a chemoconvulsant mouse model of epilepsy. iGABASnFR was engineered from bacterial periplasmic binding proteins (PBPs) by insertion of circularly permuted fluorescent proteins, and functions as a molecular switch that turns on upon GABA binding. iGABASnFR could be further used in models of epilepsy and in the cerebellum of zebrafish to help elucidate the role of GABA in various circuits, during development and in disease such as Alzheimer's disease.

https://doi.org/10.1038/s41684-019-0390-y

Alexandra Le Bras and Ellen P. Neff
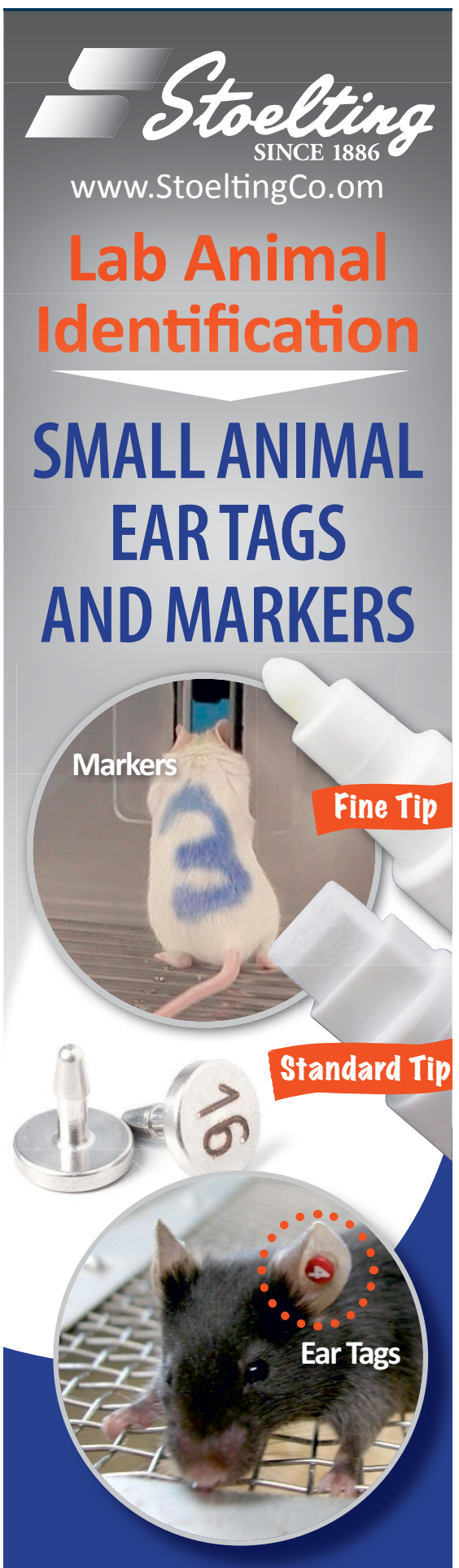

Ear Tag and Marker Features:

- Quick and easy identification

- Long-lasting

- Nontoxic, non-hazardous

- Multiple colors options available

Samples now available, Contact Stoelting for more details! info@stoeltingco.com 